\title{
Optical coherence tomography angiography characteristics in diabetic patients without clinical diabetic retinopathy
}

\author{
Ian A. Thompson ${ }^{1} \cdot$ Alia K. Durrani ${ }^{1} \cdot$ Shriji Patel $\mathbb{B}^{1}$
}

Received: 5 October 2017 / Revised: 12 January 2018 / Accepted: 14 September 2018 / Published online: 3 December 2018

(c) The Royal College of Ophthalmologists 2018

\begin{abstract}
Purpose The purpose of this study is to measure optical coherence tomography angiography (OCTA)-defined retinal vascular and choriocapillaris parameters in diabetic patients without clinically evident diabetic retinopathy.

Methods Retrospective case series of eyes in patients with a documented history of either type 1 or type 2 diabetes mellitus without any clinical signs of diabetic retinopathy seen at the Vanderbilt Eye Institute. All eyes underwent dilated funduscopic examination along with OCTA. OCTA analytics were performed to calculate vessel density, flow area, and foveal avascular zone size.

Results Thirty-seven eyes of 20 patients were included in the analysis. The mean glycated hemoglobin A1c of the 20 patients was $7.2 \%$. All patients underwent dilated funduscopic examination by a fellowship-trained retina specialist. No patients had any overt retinopathy on clinical examination. Fifteen eyes had vascular abnormalities (microaneurysms) present on OCTA. Patients with microaneurysms and patients without microaneurysms were compared. Vessel density of the $6 \times 6 \mathrm{~mm}$ macular cube images was significantly increased in patients with microaneurysms compared with patients without microaneurysms. There were no other significant differences in OCTA characteristics.

Conclusion OCTA can detect microvascular changes not otherwise noted on dilated clinical examination. These pre-clinical findings may facilitate earlier intervention for improved glycemic control and prevention of the onset of clinical retinopathy.
\end{abstract}

\section{Introduction}

Diabetic retinopathy (DR) is the leading cause of blindness in the developed world [1]. Vascular changes are among the most serious manifestations of retinal disease. Dilated fundus examination is the current gold standard in screening for vascular alterations in diabetic eye disease. Fundus photography has also proved useful as a complementary screening tool, which permits the detection of disease and monitoring of progression and treatment response from afar. Other imaging modalities such as fluorescein angiography can provide a more sensitive examination of the posterior pole; however, it is costly, time-consuming,

This data has not been presented at any national meetings or published elsewhere.

Shriji Patel

shriji.patel@vanderbilt.edu

$1 \quad$ Vanderbilt Eye Institute, Nashville, TN 37232, USA and is associated with rare but non-trivial side effects [2]. Optical coherence tomography angiography [OCTA] is a relatively new, non-invasive imaging tool that is able to provide high-resolution, well-defined images of the retinal microvasculature without the injection of dye. It works by acquiring and comparing decorrelation signals from sequential OCT B-scans at a given retinal location [3]. Background motion artifact is removed with specific software processing tools. The remaining fluctuations in OCT signal are attributed to erythrocyte movement in retinal vessels. This movement is then computed and displayed as a depth resolved map of retinal microvasculature [4].

Pre-clinical changes present on OCTA, but not on examination, have been documented in previous investigations including capillary dropout, microaneurysms, neovascularization, venous beading, and foveal avascular zone (FAZ) enlargement [5, 6]. The highly detailed visualization available on OCTA, along newer algorithms for performing higher-order vessel analytics, may permit earlier detection of other microvascular changes not otherwise seen on clinical examination. 


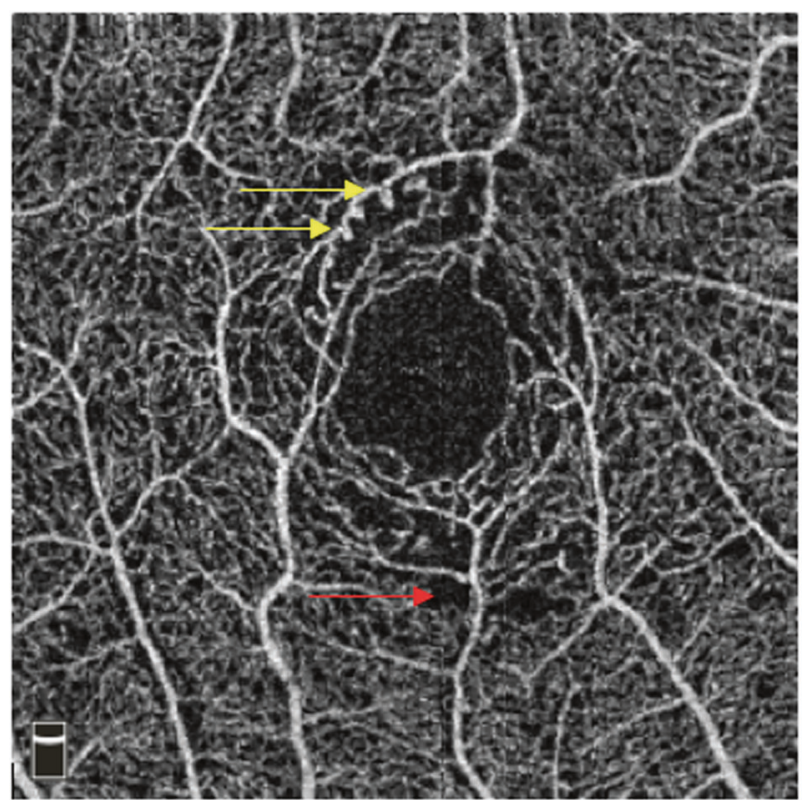

Fig. 1 OCTA showing multiple microaneurysms (yellow arrow) and areas of capillary dropout (red arrow) at the level of the superficial vascular plexus. These findings were not visible on dilated funduscopic examination

In this investigation, the authors investigate the presence of qualitative and quantitative vascular abnormalities seen on OCTA signifying diabetic retinopathy in patients without clinically evident retinopathy.

\section{Methods}

This study was performed according to the Declaration of Helsinki and was approved by the Vanderbilt University Institutional Review Board (IRB\#170783). Informed consent was obtained from all participants. A retrospective review was performed of subjects with Type 1 or Type 2 diabetes, who underwent OCTA. Inclusion criteria were age $>18$ years, history of diabetes mellitus (type 1 or 2 ) without evidence of diabetic retinopathy on dilated funduscopic examination, good quality OCTA images, and no motion artifacts to degrade interpretability of the image. Patients with history of concomitant vitreoretinal pathology or media opacity were excluded from this study. Eyes with poor-quality images on OCTA (quality index <40) were also excluded.

All subjects underwent a complete ophthalmological examination including best-corrected visual acuity testing, slit-lamp examination, and dilated fundus evaluation. Angioretina examination (Optovue, Inc., Fremont, CA, USA) was additionally performed at the time of their visit. For each patient, all analyzed images were obtained on the same day. The presence of diabetic retinopathy was based on the fundus examination using the Diabetic Retinopathy Severity Scale (DRSS) grading system [7]. All patients were classified as DRSS Level 10 (diabetic retinopathy absent). All grading was performed by experienced retinal specialists.

Patient characteristics including age, sex, duration of diabetes, most recent A1c, presence of comorbid disease including hypertension, hyperlipidemia, coronary artery disease, and/or human immunodeficiency virus (HIV) status were recorded. Comorbid disease including HIV status was obtained by self-reported history. Confirmation of HIV status by serologic test was not performed.

\section{OCTA image acquisition}

We used XR Avanti Optical Coherence Tomography Angiography (RTVue XR AVANTI, Optovue, Fremont, CA, USA) to capture images. Embedded AngioAnalytics software was used to obtain vessel density within a $6 \times$ $6 \mathrm{~mm}$ scanning area centered on the fovea (macular cube), a $3 \mathrm{~mm}$ parafoveal circle excluding the FAZ, and the fovea. En-face OCT angiograms were segmented to define the superficial capillary plexus and deep capillary plexus, using the segmentation algorithm of the built-in software. Flow area was obtained for the choriocapillaris and the outer retina. FAZ area $\left(\mathrm{mm}^{2}\right)$ was evaluated in the superficial vessel plexus by using the non-flow area tool included in the software to provide automated FAZ segmentation.

\section{Qualitative analysis}

Two different readers (AD and IT) evaluated the presence of microaneurysms on OCTA images. When available, $3 \times$ $3 \mathrm{~mm}$ OCTA images were used as an adjunct to evaluations being completed on the $6 \times 6 \mathrm{~mm}$ OCT angiograms to provide an image with smaller field of view but higher resolution. Disagreement regarding interpretation of these features was resolved by open adjudication

\section{Statistical analysis}

Statistical analysis was performed using SPSS software 22 (SPSS, Inc., Chicago, IL, USA) and GraphPad Prism 7 (Windows, GraphPad Software, La Jolla, CA, USA). Eyes were separated into groups based on the presence or absence of vascular abnormalities on OCTA and tested for normality using the Anderson-Darling normality testing. Angiographic parameters were then compared between the two groups using the two-sided Mann-Whitney $U$-test. All $P$-values were based on two- sided testing and were considered to be statistically significant if $<0.05$. 
Table 1 Quantitative comparison between groups based on presence of vascular malformations

\begin{tabular}{llll}
\hline & Microaneurysms & No microaneurysms & $P$-value \\
\hline Age (years) & $55(9.71)$ & $61.87(9.613)$ & 0.111 \\
Duration of diabetes (years) & $12.63(10.97)$ & $13.87(7.376)$ & 0.579 \\
Snellen visual acuity & $20 / 25$ & $20 / 25$ & 0.581 \\
A1C $(\%)$ & $6.7(.766)$ & $7.36(1.715)$ & 0.435 \\
Flow area - outer retina $\left(\mathrm{mm}^{2)}\right.$ & $1.116(0.251)$ & $1.172(0.1351)$ & 0.763 \\
Flow area - choriocapillaris $\left(\mathrm{mm}^{2}\right)$ & $1.887(0.094)$ & $1.864(0.099)$ & 0.339 \\
Vessel density - $6 \times 6 \mathrm{~mm}$ mac cube $(\%)$ & $49.47(4.787)$ & $45.37(4.316)$ & 0.017 \\
Vessel density - fovea $(\%)$ & $33.89(6.706)$ & $31.64(6.738)$ & 0.397 \\
Vessel density - parafovea $(\%)$ & $52.12(1.394)$ & $48.77(5.636)$ & 0.076 \\
Foveal avascular zone $\left(\mathrm{mm}^{2}\right)$ & $0.322(0.161)$ & $0.347(0.166)$ & 0.512 \\
Foveal thickness $(\mu \mathrm{m})$ & $248(30.6)$ & $256.1(27.08)$ & 0.406 \\
Parafoveal thickness $(\mu \mathrm{m})$ & $316.6(21.16)$ & $310.8(20.21)$ & 0.397 \\
\hline
\end{tabular}

\section{Results}

There were 37 eyes of 20 diabetic patients included in this study after 3 eyes were excluded for poor-quality images. There was one type 1 diabetic; the remaining were type 2 diabetes. The average age was $59.65 \pm 10.09$ years (range: 42-79). Eleven patients were women and nine patients were men. Thirteen $(56.5 \%)$ patients had concomitant hypertension, five $(65.2 \%)$ patients had coronary artery disease, five $(65.2 \%)$ patients had hyperlipidemia, and two $(8.7 \%)$ patients were HIV positive. All were found to have a normal dilated funduscopic examination by a fellowship-trained retina specialist.

This study evaluated the vasculature of diabetic eyes determined to have no diabetic retinopathy on clinical examination and found non-clinically apparent microaneurysms or capillary dropout in $8(40 \%)$ of 20 subjects on OCTA image analysis by trained readers (Fig. 1). Twelve patients were without any vascular abnormalities, confirming the findings on dilated fundus exam.

There were no demographic differences found between these two groups of patients. Vessel density in the $6 \times 6 \mathrm{~mm}$ macular cube was found to be significantly increased $(p=$ 0.017 ) in subjects with vascular abnormalities on OCTA as compared with the subjects without any evidence abnormalities on OCTA image analysis. No other statistically significant differences were noted between these two groups. See Table 1.

\section{Discussion}

Early changes in diabetic eyes start with the loss of pericytes and endothelial cells, leading to local structural weakness in the vessel wall with subsequent dilation and formation of microaneurysms [8]. Microaneurysms are therefore recognized among the first signs of clinical retinopathy. Their early recognition can help in the secondary prevention of progression of diabetic retinopathy to more severe stages through the institution of tighter glucose control and closer monitoring.

We reviewed 20 diabetic patients who were without clinical signs of diabetic retinopathy. These patients were on average 60 years old with duration of diabetes for 12-13 years. In addition, these patients had fairly good glucose control with A1c mostly in the 6-7\% range. Dilated fundus examination in these patients failed to reveal any signs of diabetic retinopathy. However, OCTA was able to demonstrate microaneurysms in $40 \%$ of our patients, suggesting that OCTA is able to detect early vascular malformations not seen on routine fundus examination. In addition, we were able to detect small areas of capillary dropout in otherwise healthy appearing retinas. This is an important finding as OCTA may be an invaluable tool in the early detection of diabetic retinopathy that is not clinically apparent.

Microaneurysms are the first clinically detectable lesions of Diabetic Retinopathy (DR) and are more likely to be observed on OCTA before they are visible on fundus examination. Their presence on OCTA demonstrates that even in diabetic patients without clinically evident retinopathy, there exists subpopulations with mild vascular abnormalities associated with impaired perfusion. Our study's novel contribution is to show that this subpopulation has a statistically significant increase in macular vessel density as defined by a $6 \times 6 \mathrm{~mm}$ field-of-view on OCTA. The clinical relevance of this information remains to be determined. However, structural abnormalities are indicators of poor capillary perfusion, and consequent ischemia of the retinal parenchyma. This tissue hypoxia increases expression of vascular endothelial growth factor (VEGF), which promotes angiogenic responses and vascular permeability, and leads to proliferative diabetic retinopathy and 
diabetic macular edema. We speculate that increased vessel density may reflect worsening subclinical disease in diabetic patients with "healthy" eyes and may represent the first sequelae of underlying retinal/choroidal tissue hypoxia.

VEGF is a known mediator of hypoxia induced inflammation and is associated with vascular remodeling in DR. Previous reports have demonstrated an association between level of aqueous VEGF and severity of DR [9]. Optic disc neovascularization, neovascularization of the iris, and neovascularization elsewhere are all thought to have their etiology in the upregulation of local VEGF production in response to worsening retinal or choroidal ischemia. Increased vessel density may be acting as a surrogate marker for locally upregulated VEGF. If OCTA can detect vascular changes before the occurrence of clinically detectable disease, and these changes are associated with increased retinal vessel density and possibly elevated VEGF, OCTA may allow us to better identify diabetic patients without DR most likely to progress. Longitudinal studies would be helpful in determining whether the observed subclinical variations in retinal vessel density and appearance have prognostic utility.

The role of antiangiogenic agents in the management of DR has recently been expanded to include non-proliferative DR [10]. The cost of anti-VEGF therapy is an important factor in its implementation. OCTA may help us better define those individuals most likely to benefit from antiVEGF treatment, presumably those diabetic subjects without DR, who have microaneurysms and increased retinal vessel density. Larger longitudinal study would confirm more conclusively whether vessel remodeling and other vascular morphologic changes precede the appearance of vascular events, such as frequency of microaneurysms and hemorrhagic leakage, or are a consequence of these events.

There were several limitations to this study. The sample size and retrospective nature of our analysis limit the generalizability of our findings. Second, our external validity is partly challenged by the setting of our study. Our ultimate target population is the group of patients with diabetes but no diabetic retinopathy. Our study patients were all referred to the Vanderbilt Eye Clinic for diabetic eye screening. These patients differ from our larger target population in two important ways. (1) The study patients were compliant with the request to be seen at the Vanderbilt Eye Clinic for routine screening and are likely more interested in their health than the general target population. (2) The percentages of comorbid disease in our study suggest that our cohort may be less healthy than the general diabetic population without diabetic retinopathy. Consequently, our estimates on the prevalence of pre-clinical vascular changes cannot be considered definitive. Lastly, statistical significance was not adjusted to account for correlations between two eyes from the same individual, because the comparisons in this study were exploratory. Even with these limitations, the findings of our present study show that diabetic eyes without clinically evident diabetic retinopathy have early vascular changes, mainly microaneurysms, which can be appreciated on OCTA. In addition, diabetic eyes without diabetic retinopathy but visible microaneurysms on OCTA have statistically significant increased macular vessel density compared with diabetic eyes without DR and without angiographically evident microaneurysms.

In summary, impaired capillary perfusion in diabetic patient without DR can be objectively characterized using OCTA. Our results suggest that vessel density in the central macula may be able to differentiate diabetic eyes with early microvasculature changes from diabetic eyes without early microvasculature changes. These findings may be a surrogate for early upregulation of VEGF. OCTA may help facilitate earlier intervention by characterizing subclinical disease. This is the subject of ongoing investigation. Measurements based on OCTA may have value in the management and diagnosis of diabetes even in patients without DR.

\section{Summary}

\section{What was known before}

- Until now, the gold standard for detection of diabetic retinopathy is dilated fundoscopic examination.

\section{What this study adds}

- OCT angiography can allow for preclinical detection of diabetic retinopathy even before evidence on dilated retina examination. This imaging technique can be used to identify and treat patients earlier on in the course of their diabetic eye disease.

Funding This study was supported by a grant from Research to Prevent Blindness.

\section{Compliance with ethical standards}

Conflict of interest The authors declare that they have no conflict of interest.

\section{References}

1. Prokofyeva E, Zrenner E. Epidemiology of major eye diseases leading to blindness in Europe: a literature review. Ophthalmic Res. 2012;47:171-88.

2. Gass JD, Sever RJ, Sparks D, Goren J. A combined technique of fluorescein funduscopy and angiography of the eye. Arch Ophthalmol. 1967;78:455-61. 
3. de Carlo TE, Bonini Filho MA, Chin AT, et al. Spectral-domain optical coherence tomography angiography of choroidal neovascularization. Ophthalmology. 2015;122:1228-38.

4. Matsunaga D, Yi J, Puliafito CA, Kashani AH. OCT angiography in healthy human subjects. Ophthalmic Surg Lasers Imaging Retin. 2014;45:510-5.

5. Hwang TS, Jia Y, Gao SS, et al. Optical coherence tomography angiography features of diabetic retinopathy. Retina. 2015;35: 2371-6.

6. Ishibazawa A, Nagaoka T, Takahashi A, et al. Optical coherence tomography angiography in diabetic retinopathy: a prospective pilot study. Am J Ophthalmol. 2015;160:35-44 e31.
7. Wilkinson CP, Ferris FL 3rd, Klein RE, et al. Proposed international clinical diabetic retinopathy and diabetic macular edema disease severity scales. Ophthalmology . 2003;110:1677-82.

8. Cai J, Boulton M. The pathogenesis of diabetic retinopathy: old concepts and new questions. Eye (Lond). 2002;16: 242-60.

9. Qaum T, Xu Q, Joussen AM, et al. VEGF-initiated blood-retinal barrier breakdown in early diabetes. Invest Ophthalmol Vis Sci. 2001;42:2408-13.

10. LUCENTIS [package insert]. South San Francisco, CA: Genentech, Inc.; 2017. http://www.lucentis.com/hcp.html. Accessed 5 June 2017. 